\title{
Lidocaine 5\% spray instillation for post-extubation laryngeal spasm management following minor ENT procedures
}

Papaioannou E.,_Bartzi K., Bakalaki V., Kavezou F., Lambadariou A., Tsinari K.

Laikon General Hospital of Athens, Department of Anaesthesiology, Athens, Greece

\section{Background - Aim}

Post-extubation laryngeal spasm is a rather common complication following ENT procedures. On a prospective study in a course of 15 years we studied 1879 patients aged 15-64, who underwent minor surgical procedures, such as amygdalectomy, microlaryngoscopy, adenotomy, sinusotomy, and nasal septopasty or polypectomy.

\section{Material-Methods}

Anaesthetic protocol was the same for all studied patients. Ranitidine 50mg and dexamethasone $8 \mathrm{mg}$ iv were administered preoperatively as gastric protection ant antiemesis, anaesthesia was induced by midazolam $0.03-0.05 \mathrm{mg} / \mathrm{kg}$ and propofol $1.5 \mathrm{mg} / \mathrm{kg}$, muscle paralysis was achieved by cis-atracurium $0.15 \mathrm{mg} / \mathrm{kg}$ and analgesic needs were covered by $2-4 \mu \mathrm{g} / \mathrm{kg}$ accordingly. Mean operation duration was $38 \pm 6,5 \mathrm{~min}$. Most patients $1765(93.9 \%)$ received clonidine $1.5 \mu \mathrm{g} / \mathrm{kg}$ iv as hypotensive agent to control excessive bleeding. Paracetamol $1 \mathrm{gr}$ and lornoxicam $8 \mathrm{mg}$ iv were administered to all patients as postoperative analgesia. For muscle paralysis reversal patients were given atropine $(0.014 \mathrm{mg} / \mathrm{kg})$ and neostigmine $(0.05-0.0 .08 \mathrm{mg} / \mathrm{kg})$. Among studied patients, 341(3,72\%) demonstrated post-extubation inspiratory stridor, $\mathrm{SpO} 2$ decrease and agitation which indicated laryngospasm. Drug allergies and vocal cord dysfunction due to residual muscle paralysis were excluded.

\section{Results}

In all patients slight positive pressure ventilation with face mask 100\% O2 was applied and lidocaine 5\% spray (5-10 times)was intrabuccally administered. In 306 (89.7\% among patients who developed stridor), signs of laryngeal spasm improved and they did not need any other medication. In 39 patients $(11.43 \%)$, lidocaine $1.5 \mathrm{mg} / \mathrm{kg}$ and diazepam $0.04-0.08 \mathrm{mg} / \mathrm{kg}$ iv were administered in order to manage laryngospasm and none of the patients needed re-intubation. All patients did not seem to remember anything afterwards and their overall satisfaction was high.

\section{Conclusion}

A few publications report laryngeal spasm, or bronchospasm improvement by lidocaine used in aerosol mask, but there are no data about using lidocaine spray directly intrabuccally in post extubation laryngospasm. 